\title{
Implementation of variable segments to model the arterial system using electromechanical analogy
}

\author{
Stefan Borik, Ivo Cap, Branko Babusiak, Klara Capova *
}

\begin{abstract}
The article deals with the design of an electrical model of variable segments of a non-symmetrical tree of small arteries This model can be used to simulate the blood pressure and flow. Peripheral arterial resistance changes are modelled by an exponentially dependent resistor. By modulating the capacitor value, we can model the arterial wall properties which depend on the arterial pressure. Simulations are performed in which vasoconstriction and vasodilation were modelled by varying the transmural pressure. As a result, we can observe the changes in the blood pressure for each arterial generation.
\end{abstract}

K e y w o r d s: arterial system, electromechanical analogy, modelling and simulations, small arteries, transmural pressure

\section{Introduction}

When compared with large arteries, small arteries and arterioles have similar physical properties from the viewpoint of blood pressure and blood flow. The basic difference rests in their spatial organization. The mother vessel can bifurcate to two daughter vessels. This branching pattern can be observed through the whole arterial tree. In addition, we find the difference in the vessel length-todiameter ratio. This ratio is relatively high in the case of large arteries and, on the other hand, for small arteries this ratio is small as their lengths and diameters are comparable $[1,2]$. We used a model of the arterial tree with distributed parameters which is based on the use of electromechanical analogy. Consequently, we used the transmission line theory and in this way, it is possible to divide the model of the arterial tree to appropriately long segments with specific geometrical and mechanical properties. Spatial organization was adopted from [3]. The single segment is shown in Fig. 1, where resistance $R$ corresponds to blood viscosity, inductance $L$ corresponds to blood density and capacitance $C$ is connected to the arterial wall elasticity $[3-6]$.

\section{Materials}

There are many regulation mechanisms of the arterial system. Branching of small arteries offers an extension of this system by implementing such a regulation mechanism which is connected to the changes of arterial radii. It is peripheral vascular resistance regulation which can be implemented to the model of the arterial system by modification of the original small arterial segment, see Fig. 1. The modification is based on the elements with variable parameters. These parameters change with alternation of the internal and external stimuli. The arterial pressure and flow vary depending on the arterial radius, and the arterial wall properties such as arterial elasticity are sensitive to the arterial radius changes. Therefore, it is needed to modify the original arterial segment to a segment with variable resistor $R_{1}$ and variable capacitor $C$. By modulating the resistor value $R_{1}$ one can model the peripheral arterial resistance changes, while by alternating the capacitance $C$ relates to the arterial wall properties which are a function of arterial pressure.

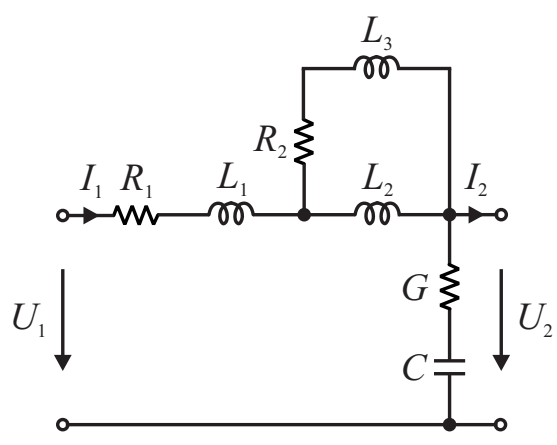

Fig. 1. Arterial segment transformed to an electrical circuit using electromechanical analogy

By using the Ohm law we get the equation for the voltage across capacitor $C$

$$
\frac{\mathrm{d} u_{C}(t)}{\mathrm{d} t}=\frac{1}{C(u(t))} i(t)
$$

But for our implementation, the integral form of (1) is preferable

$$
u_{C}(t)=\int \frac{1}{C(u(t))} i(t) \mathrm{d} t
$$




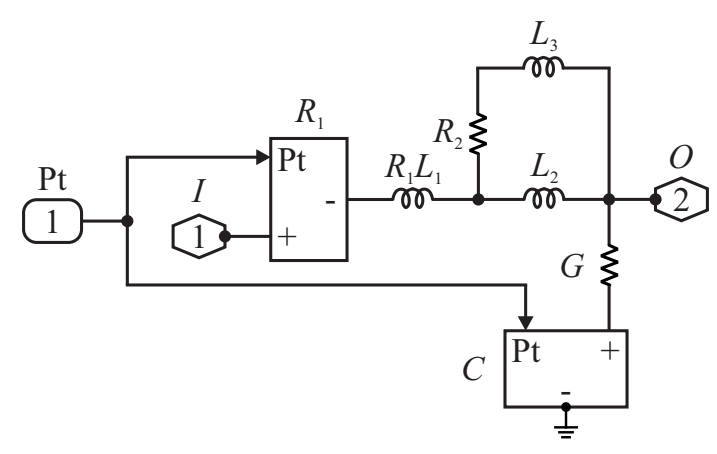

Fig. 2. Arterial segment with variable elements implemented in SIMULINK

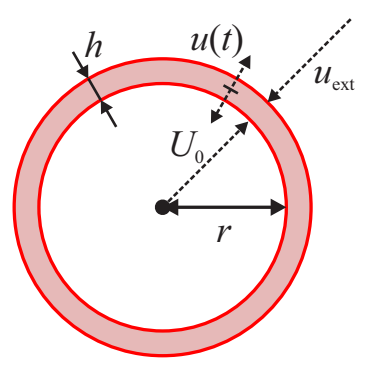

Fig. 3. Pressures acting on the arterial wall

where $i(t)$ is the electrical current flowing through the transversal branch of the modelled arterial segment and $u_{C}(t)$ is the electrical voltage across capacitor $C$ (see Fig. 1 or its modified version Fig. 2).

In our implementation, we use voltage $u(t)$ which represents the transmural pressure from the point of view of the electromechanical analogy. Transmural pressure $u(t)$ is defined by subtraction of the mean pressure $U_{0}$ inside the artery and the external pressure $u_{\text {ext }}(t)$ caused by some regulation mechanism or striated muscle contraction. These pressures have an antagonistic effect and their resultant is the transmural pressure which relates to the transversal dilatation or constriction of the selected artery, see Fig. 3,

$$
u(t)=U_{0}-u_{\text {ext }}(t) .
$$

By modelling the transmural pressure changes we can simulate the arterial constriction $(u(t)<0)$ or arterial dilatation, where $u(t)>0$. The values of elements $R_{1}$ and $C$ vary depending on the voltage $u(t)$. We can define a change in capacitance $C$

$$
C(u)=C_{0} \mathrm{e}^{-\alpha_{C} \frac{u(t)}{U_{0}}}
$$

where $C_{0}$ is the initial value of capacitance when we assume the artery in the initial condition (internal and external pressures equal and it follows that $u(t)=0$ ). Using the parameter $\alpha_{C}$ it is possible to change the curve slope characterizing the capacitance value in dependence on the transmural pressure changes. In this way we can model the arterial wall response to pressure changes. The voltage across capacitor $C$ is

$$
\begin{aligned}
& u_{C}(t)=\frac{1}{C_{0}} \int i(t) \mathrm{e}^{\alpha_{C} \frac{u(t)}{U_{0}}} \mathrm{~d} t= \\
& \frac{1}{C_{0}} \int i(t) \mathrm{e}^{\alpha_{C}\left(1-\frac{u_{\mathrm{ext}}(t)}{U_{0}}\right)} \mathrm{d} t .
\end{aligned}
$$

We can use an analog derivation also to model the variable resistor $R_{1}$. When the artery constricts, the $R_{1}$ value increases and vice versa, so the voltage across the resistor is

$$
u_{R_{1}}(t)=R_{1}(u(t)) i_{1}(t)
$$

where $i_{1}(t)$ represents the electrical current flowing through resistor $R_{1}$ whose value is defined similar to (6)

$$
R_{1}=R_{0} \mathrm{e}^{-\alpha_{R} \frac{u(t)}{U_{0}}}
$$

where $R_{0}$ is the value of the peripheral vascular resistance of the selected arterial segment in the initial state (internal and external pressures equal and it follows that $u(t)=0)$. Using the parameter $\alpha_{R}$ it is possible to change the curve slope characterizing the resistance value in dependence on the transmural pressure changes and in this way we can model the arterial wall response to the pressure changes. The voltage across resistor $R_{1}$ is expressed as

$$
u_{R_{1}}(t)=R_{0} \mathrm{e}^{-\alpha_{R} \frac{u(t)}{U_{0}}} i_{1}(t)
$$

Figure 4 shows the capacitance and resistance value changes in dependence on the different values of the transmural pressure. Dilating the artery, the fibres which the artery consists of are stretched and in this way the artery becomes more rigid. Its compliance or elasticity decreases and its overall capacitance and resistance also decrease. By constriction of the artery, the overall resistance increases and by dilatation the resistance decreases. Variable segment according to Fig. 4 are placed in the tree of the small arteries in the fifth generation on the left side, see [7].

\section{Results}

Functionality of the variable element segments was verified in the MATLAB-SIMULINK programming environment. Variable segments were placed in the $4^{\text {th }}$ and $5^{\text {th }}$ generations of the modelled arterial tree. We measured the voltage at the place of each bifurcation until the $7^{\text {th }}$ generation in the $\beta$ side of the tree (see [7] for topology details. During simulation, the transmural pressure was changed from -100 to $100 \mathrm{mmHg}$ by using

$$
u(t)=U_{\mathrm{m}} \sin (2 \pi f t)
$$

where $U_{\mathrm{m}}$ represents the amplitude of the signal, $f$ is oscillation frequency of the transmural pressure set to $0.1 \mathrm{~Hz}$ and $t$ is time in seconds.

By using (8) it is possible to simulate the changes in the small arterial tree. As discussed in the text above, 

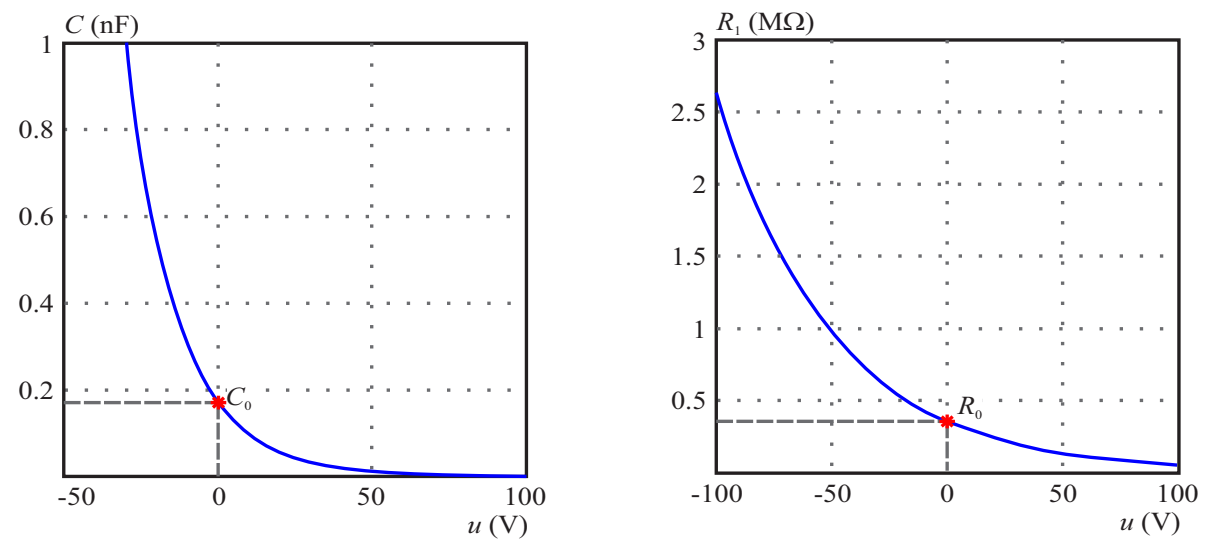

Fig. 4. Capacitance $C$ (left) and resistance $R_{1}$ (right) changes in dependence on the transmural pressure

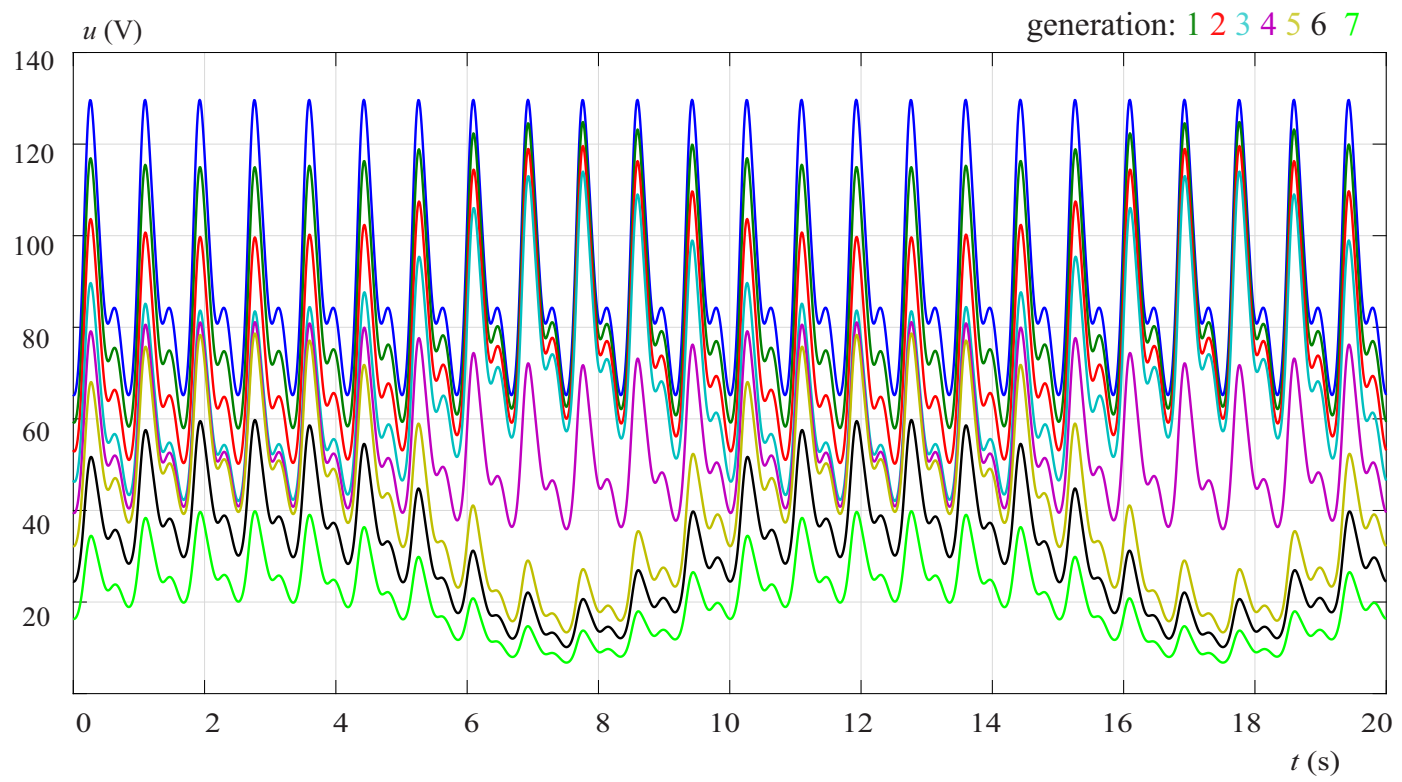

Fig. 5. Simulation results of transmural pressure changes and their impact on the blood pressure in small arterial segments

constriction occurs at $u(t)<0$. Vasoconstriction is visible, eg, from the $5^{\text {th }}$ to the $10^{\text {th }}$ second of the simulated arterial pressure in the appropriate generations, see Fig. 5. We can see a blood pressure drop by the increased peripheral resistance simulated by resistor $R_{1}$. We can observe the impact of the arterial elasticity changes modelled by capacitor $C$ at the 8 th second of the record. The dicrotic notch of the pressure curve is flatter and this phenomenon is caused by capacitance changes which have an impact on the cutoff frequency of the selected segment. By simulation of the variable elements we can observe the pressure and flow changes in single small arterial segments in appropriate generations.

\section{Conclusions}

A tree of small arteries was created per physiological observations described in [8-12]. Placing of variable elements into single arterial segments leads to pressure (voltage) changes in the small arterial tree. The presented model can serve for examination and observation of the pressure and flow changes related to certain regulation mechanisms causing vasomotion etc. There is a possibility to modulate the arterial dilatation or constriction easily by changing the parameters of exponential functions which describe the behaviour of the variable elements. Modelling of the vascular system by electromechanical analogies can lead to a better understanding of the processes which occur in arteries. Also, it could be possible to evaluate the degree of pathological changes in the arterial system by using appropriate measurement methods (eg, photoplethysmography) [12] and by reverse comparisons of the measured and simulated data. In this way, malformations such as arterial stenosis or aneurysm could be deeply studied.

\section{REFERENCES}

[1] A. S. Iberall, "Anatomy and Steady Flow Characteristics of the Arterial System with an Introduction to its Pulsatile Characteristics", Mathematical Biosciences, 1(3), 375-395, 1967. 
[2] B. J. West, V. A. L. M. I. K. Bhargava, and A. L. Goldberger, "Beyond the Principle of Similitude: Renormalization the Bronchial Tree", Journal of Applied Physiology, 60(3), 1089-1097, 1986

[3] A. P. Avolio, "Multi-Branched Model of the Human Arterial System", Medical and Biological Engineering and Computing, 18(6), 709-718, 1980.

[4] J. R. Womersley, "An Elastic Tube Theory of Pulse Transmission and Oscillatory Flow Mammalian Arteries", Aerospace Research Labs Wright-Patterson AFB OH, 1957.

[5] B. Czippelova and D. Gombarska, "Modelling of Arterial Bifurcation by Means of Electromechanical Model with Distributed Parameters", Komunikacie 13(1), 22-26, 2011.

[6] E.W.Gaelings, Numerische simulation hämodynamischer prozesse vaskulären Netzen Shaker, 1996.

[7] S. Borik, I. Cap and B. Babusiak, "Analysis of Nonsymetrical Arterial Branching using Electromechanical Analogy", ELEKTRO, 2014. IEEE, 522-525, 2014.

[8] A. Guyton, and J. Hall, Textbook of Medical Physiology Elsevier, Philadelphia, 2006.

[9] M. S. Olufsen, C. S. Peskin, W. Y. Kim, E. M. Pedersen, A. Nadim and J. Larsen, "Numerical Simulation and Experimental Validation of Blood Flow Arteries with Structured-Tree Outflow Conditions", Annals of biomedical engineering 28(11), 1281-1299, 2000.

[10] C. D. Murray, "The Physiological Principle of Minimum Work: I. the Vascular System and the Cost of Blood Volume", Proceedings of the National Academy of Sciences of the United States of America 12(3), 207, 1926.

[11] M. S. Olufsen, "Structured Tree Outflow Condition for Blood Flow Larger Systemic Arteries", American journal of physiology-Heart and circulatory physiology 276(1), H257-H268, 1999.

[12] V. Blazek, "Biomedical Technology - 2011 and beyond", Komunikacie 13(1), 5-12, 2011.

Received 22 March 2017

Štefan Borik was born in 1986. He received his MSc degree in 2011 and $\mathrm{PhD}$ degree in 2014 from the Faculty of Electrical Engineering, Department of Electromagnetic and Biomedical Engineering, University of Žilina. Currently, he works as an assistant professor at the Department of Electromagnetic and Biomedical Engineering, University of Žilina. He especially deals with analog and digital hardware design, biological signal processing and modelling.

Ivo Čáp was born in 1948. He graduated from the Czech Technical University in Prague, Faculty of Electrical Engineering, received the $\mathrm{PhD}$ degree from the Slovak Academy of Sciences, Institute of Electrical Engineering, and was appointed professor of condensed matter physics and acoustics at the University in Žilina. The present appointment is professor of biomedical engineering at the Faculty of Electrical Engineering of the University of Žilina. He is specialized in modelling of physiological processes, of the influence of EM field on living organisms and on EM non-destructive testing of conducting materials.

Branko Babušiak was born on 17 January 1983. In 2006, he graduated (MSc) from the Department of Electromagnetic and Biomedical Engineering of the Faculty of Electrical Engineering at University of Žilina. He received his $\mathrm{PhD}$ in the field of technical cybernetics in 2009 at the Department of Measurement and Control of the Faculty of Electrical Engineering and Computer Science at VSB-Technical University of Ostrava. Since 2009 he has worked as a tutor with the Department of Electromagnetic and Biomedical Engineering in Žilina. His scientific research is focused on digital signal processing of biological signals and biomedical image processing.

Klára Čápová received her MSc degree from the Faculty of Electrical Engineering, Czech Technical University in Prague in 1971 and PhD degree from the Faculty of Electrical Engineering and Information Technology, Slovak Technical University in Bratislava in 1981. Since 2001 she has been professor and head of the Department of Electromagnetic Engineering and since 2005 up to now at the Department of Electromagnetic and Biomedical Engineering, Faculty of Electrical Engineering, University of Žilina. The main area of her research and teaching activities has moved from the electrical circuits and electromagnetic field theory and its application, mainly electromagnetic methods for non-destructive evaluation, to biomedical engineering. 